Edited by Kiriakos Xenitidis and
Colin Campbell
Contents
- Link between outcome and service quality
is not clear
- Missing ethnic density data
- in addiovascular fitness and serious depression
- New paradigm: developmental psychopathology

\section{Link between outcome and service quality is not clear}

In their study of rehabilitation services in England, Killaspy and co-authors conclude that 'Quality of care is linked to better clinical outcomes in people with complex and longer-term mental health problems.' ${ }^{1}$ We are not persuaded that this conclusion is justified by the results reported.

The study investigated the relationship between measures of quality and clinical outcomes across a number of services at different locations in England. The scales used to measure outcomes consisted of the Resident Choice Scale (RCS), the Manchester Short Assessment of Quality of Life (MANSA), the Your Treatment and Care (YTC), the General Milieu Index (GMI) and the Global Assessment of Functioning (GAF).

Our main argument is that of these the RCS, YTC and GMI are actually measures of the service user's perception of the quality of care rather than measuring what most people would understand by the term 'clinical outcome'. Although these measures are widely referred to as outcome measures, we argue that the term 'clinical outcome' should refer to factors such as symptom level, relapse rates, functioning, carer burden and quality of life. The RCS, YTC and GMI scales were reported to be associated with the objective measures of service quality. However, we would argue that this result simply demonstrates that patients are able to make a valid judgement about the quality of the services they receive.

On the other hand, we agree that the MANSA and GAF are indeed measures of clinical outcome. However, the MANSA, which does measure quality of life, did not show any association with measures of service quality and the authors did not report any results for the GAF.

Based on these findings we are unable to agree with the authors' conclusion that they have demonstrated an association between service quality and clinical outcomes. This negative finding is aligned with that of a study that they cite and which failed to demonstrate an improvement in outcomes following the implementation of the quality and outcomes framework for diabetes. ${ }^{2}$

It seems that it is difficult to convincingly demonstrate a real relationship between care quality and clinical outcomes. However, we would argue that attempts to do this should use outcome measures of real relevance, such as the Health of the Nation Outcome Scales (HoNOS), ${ }^{3}$ GAF and MANSA.

1 Killaspy H, Marston L, Omar RZ, Green N, Harrison I, Lean M, et al. Service quality and clinical outcomes: an example from mental health rehabilitation services in England. Br J Psychiatry 2013; 202: 28-34.

2 Calvert M, Shankar A, McManus R, Lester $H$, Freemantle N. Effect of the quality and outcomes framework on diabetes care in the United Kingdom: retrospective cohort study. BMJ 2009; 338: b1870.
3 Wing JK, Beevor AS, Curtis RH, Park SB, Hadden S, Burns A. Health of the Nation Outcome Scales (HoNOS). Research and development. Br J Psychiatry 1998; 172: 11-8.

Prince Arun Vincent, East London NHS Foundation Trust, Glasshouse Fields Centre, 68 Glasshouse Fields, London E1W 3AB, UK. Email: princeavincent@gmail.com; David Curtis, Glasshouse Fields Centre, East London NHS Foundation Trust, UK

doi: 10.1192/bjp.202.4.309

Authors' reply: On the basis of their definition of outcome, Vincent \& Curtis challenge the conclusions of our national study of mental health in-patient rehabilitation services where we found that the quality of services was positively associated with better clinical outcomes. In doing so, they seem to adopt a medical model and ignore important insights arising from patient-centred concepts of recovery. They regard 'symptoms, functioning and relapse' as key, whereas a recovery-oriented approach would also value the quality of therapeutic relationships, the promotion of autonomy and better quality of life.

Our aim was to try to bridge this ideological divide by placing patient-reported outcomes on an equal footing with so-called clinical measures. At the risk of excessive pedantry, it would be fair to say that measures of patient experiences of care, such as those we used in our study, could be considered assessments of process rather than outcome, although they are nevertheless an important aspect in the measurement of service quality. In any case, such measures are usually referred to as patient-reported outcome measures. We found a positive association between our service managers' assessments of the quality of their own services (using our standardised measure, the Quality Indicator for Rehabilitative Care, QuIRC) and patients' experiences of care. This corroborates the service managers' ratings and strongly suggests that improving service quality will result in a better service user experience surely an 'outcome' everyone can relate to as worthwhile.

We also found that greater quality of mental health rehabilitation services was associated with greater service user autonomy. We gave a number of possible reasons why we did not find a positive association between service quality and service user quality of life, the main one being that the measure we used focuses on experiences outside of an in-patient setting (relationships with family/partner, work, income, etc.). Our findings reflect the reality for people in in-patient mental health rehabilitation units who tend to have lengthy admissions (in our study, their current admission was 18 months on average, with 8 of these in the rehabilitation unit) due to the severity and complexity of their symptoms and severe impairment of social functioning, all of which have a negative impact on their social inclusion and quality of life. Nevertheless, it is absolutely correct that rehabilitation services should (and do) aim to help service users achieve a successful community life which, ultimately, should be reflected in their quality of life.

Our findings reflect the focus of our study - in-patient mental health rehabilitation services deal with individuals at the beginning of their rehabilitation, when they are most severely unwell and least able to engage in the community. Later phases of our research will provide further data on the longitudinal outcomes, including social functioning and successful community living.

Helen Killaspy, Mental Health Sciences Unit, University College London, Charles Bell House, 67-73 Riding House Street, London W1W 7EJ, UK. Email: h.killaspy@ucl.ac.uk; Louise Marston, Department of Primary Care and Population Health, Rumana Z Omar, Department of Statistical Science, Nicholas Green, Isobel Harrison, Melanie Lean, Mental Health Sciences Unit, University College London; Frank Holloway, South London and Maudsley NHS Foundation Trust; Tom Craig, Health Services Research Department, Institute of Psychiatry, King's College London, and South London and Maudsely NHS Foundation Trust; Gerard Leavey, Northern Ireland Association of Mental Health, Belfast; Michael King Mental Health Sciences Unit, University College London, and Camden and Islington NHS Foundation Trust, UK

doi: $10.1192 /$ bjp.202.4.309a 